\title{
1 Antibiotic degradation by commensal microbes shields pathogens
}

2 Mergim Gjonbalaj ${ }^{1}$, James W. Keith ${ }^{1,2}$, Mytrang Do ${ }^{1,3}$, Tobias M. Hohl ${ }^{1,4}$, Eric G. Pamer ${ }^{1, \#}$,

3 Simone Becattini ${ }^{1, \S}$

41 Immunology Program, Sloan Kettering Institute, Memorial Sloan Kettering Cancer Center, New

5 York, NY, USA

62 Louis V. Gerstner Jr. Graduate School of Biomedical Sciences, Memorial Sloan Kettering Cancer

7 Center, New York, NY, USA

83 Weill Cornell Medical College, New York, NY, USA

94 Infectious Disease Service, Department of Medicine, Memorial Hospital, Memorial Sloan

10 Kettering Cancer Center, New York, NY, USA

11 \# current address: The Duchossois Family Institute, University of Chicago, Chicago, IL, USA

$12 \S$ corresponding author

14 Abstract

15 The complex bacterial populations that constitute the gut microbiota can harbor antibiotic-

16 resistance genes (ARGs), including those encoding for $\beta$-lactamase enzymes (BLA), which

17 degrade commonly prescribed antibiotics such as ampicillin. While it is known that ARGs can be

18 transferred between bacterial species, with dramatic public health implications, whether

19 expression of such genes by harmless commensal bacterial species shields antibiotic-sensitive

20 pathogens in trans by destroying antibiotics in the intestinal lumen is unknown. To address this

21 question, we colonized GF mice with a model intestinal commensal strain of $E$. coli that

22 produces either functional or defective BLA. Mice were subsequently infected with Listeria 
23 monocytogenes or Clostridioides difficile followed by treatment with oral ampicillin. Production

24 of functional BLA by commensal E. coli markedly reduced clearance of these pathogens and

25 enhanced systemic dissemination during ampicillin treatment. Pathogen resistance was

26 independent of ARG acquisition via horizontal gene transfer but instead relied on antibiotic

27 degradation in the intestinal lumen by BLA. We conclude that commensal bacteria that have

28 acquired ARGs can mediate shielding of pathogens from the bactericidal effects of antibiotics.

\section{Importance}

31 The wide use of antibiotics in human populations and in livestock has led to increasing

32 prevalence of pathogenic and commensal bacterial species that harbor antibiotic resistance

33 genes (ARGs), such as those encoding for ampicillin-degrading $\beta$-lactamases. We investigated

34 whether harmless autochthonous bacteria might degrade orally administered antibiotics,

35 thereby impairing their ability to combat intestinal pathogens. Here we report that antibiotic

36 degradation by a resident intestinal strain of $E$. coli reduces the effectiveness of oral ampicillin

37 against two intestinal pathogens, L. monocytogenes and C. difficile, resulting in increased

38 intestinal and systemic bacterial burden. We demonstrate that expression of ARGs by non-

39 pathogenic members of the gut microbiota shields antibiotic-sensitive pathogens and enhances

40 their expansion and dissemination.

41

42

\section{Observation}


Antibiotic administration has markedly reduced the morbidity and mortality associated with bacterial infections in the pre-antibiotic era. Increasing antibiotic-resistance in pathogenic microbes, mediated in part by acquired genes that encode antibiotic-degrading enzymes,

47 represents a major threat to human health (1). The gut microbiota contains trillions of commensal bacteria that can also harbor

49 antibiotic resistance genes (ARGs) (2). Notably, antibiotic exposure can increase ARG gene 50 representation and expression by the gut microbiota (3). Horizontal ARG transfer represents a 51 mechanism by which drug-sensitive microbes can acquire resistance, e.g. by acquisition of 52 genes encoding antibiotic-degrading hydrolases $(4,5)$. Thus, it is possible that commensal

53 bacterial species transfer ARGs to intestinal pathogens upon antibiotic exposure in the gut

54 lumen. However, another possibility is that production of antibiotic-degrading enzymes by the

55 resident microbiota protects otherwise drug-sensitive pathogens in trans, thereby facilitating

56 their replication and spread in the host.

To test this hypothesis in a controlled system, we reconstituted germ-free (GF) mice

58 with an E. coli strain, utilized here as a model commensal, that expresses either a WT form of $\beta$ -

59 lactamase (TEM-1) or an inactive point mutant (hereby referred to as WT BLA or mut BLA,

60 respectively) (Figure $1 A)(6)$. This approach yielded cohorts of mice that, with the exception of

61 one codon, harbor identical genomes, thus excluding differences in microbiota functions (e.g.,

62 immune activation, colonization resistance, etc.) that are not related to the $\beta$-lactam

63 degradation.

64 Although WT BLA and mut BLA E. coli reached identical luminal bacterial densities in 65 reconstituted mice, a colorimetric assay confirmed that only the intestinal content of mice 
reconstituted with WT BLA E. coli retained the capacity to hydrolyze $\beta$-lactams (Figure $1 \mathrm{~B}, \mathrm{C}$ ).

67 One week after reconstitution, mice were orally infected with the foodborne pathogen Listeria monocytogenes $10403 \mathrm{~s}(\mathrm{Lm})$. $\mathrm{Lm}$ is highly sensitive to $\beta$-lactam antibiotics and can expand in

69 the gut lumen of mice that lack colonization resistance (7). Mice were then administered

70 ampicillin on day +1 and +2 post $L m$ infection and sacrificed on day +3 . As expected, $L m$ reached

71 identical densities in the intestines of WT BLA or mut BLA E. coli reconstituted mice on day +1 ,

72 indicating that the 2 E. coli strains did not differ in their inability to provide colonization

73 resistance against $L m$. However, we found significantly higher $L m$ burden in multiple organs in

74 mice harboring WT BLA E. coli on day +3 , consistent with the notion that $\beta$-lactamase-

75 dependent ampicillin degradation shielded $L m$ from the therapeutic antibiotic's action.

76 To exclude the possibility that $L m$ might have acquired resistance to ampicillin via horizontal

77 gene transfer, we inoculated single $L m$ colonies recovered from the cecal content of WT BLA E.

78 coli-reconstituted, $L m$-infected mice, into liquid medium either in the presence or absence of

79 ampicillin. Notably, none of the inoculated $\mathrm{Lm}$ colonies grew in the presence of ampicillin, in

80 contrast to WT BLA-expressing E. coli colonies recovered from the same mice (Figure 1F).

81 Furthermore, none of the $L m$ colonies tested positive for the presence of the $\beta$-lactamase gene,

82 which was uniformly detected in colonies of WT BLA E. coli by PCR (Figure 1G).

To confirm that the increased $L m$ burden observed above was due to antibiotic

84 degradation by resident $E$. coli, we collected the cecal contents of mice reconstituted with

85 either WT BLA or mut BLA E. coli and treated with ampicillin in the drinking water for two

86 consecutive days to allow for luminal accumulation of the antibiotic. Inoculation of $L m$ into

87 serial dilutions of the cecal content supernatants revealed that the cecal contents from mice 
reconstituted with mut BLA E. coli had a higher inhibitory capacity compared to cecal contents recovered from mice reconstituted with WT BLA E. coli (Figure $1 \mathrm{G}$ and Supplementary Figure 1). Since the presence of active $\beta$-lactamase was the only bona fide difference between the cecal

91 contents of the two cohorts of mice, we conclude that the microbiota-encoded enzymatic

92 activity curtailed the efficacy of ampicillin treatment against $\mathrm{Lm}$.

94 To expand our observations beyond the Listeria model, and to assess whether commensal-

95 mediated antibiotic degradation may represent a mechanism that is relevant to other infectious

96 agents, we adapted our experimental strategy to an established $C$. difficile infection model (8)

97 (Figure 2A), an important intestinal pathogen that is also sensitive to ampicillin (Supplementary

98 Figure 2). Of note, this model allowed us to investigate the relevance of our findings in a setting

99 where expansion of an antibiotic-resistant microbe takes place following antibiotic-mediated

100 depletion of the intestinal microbiota, a common occurrence in hospitalized patients (9).

101 Similar to the results obtained with $\mathrm{Lm}$, we observed indistinguishable levels of expansion for

102 both $E$. coli and $C$. difficile on day +1 after reconstitution or infection, respectively, in all groups

103 of mice (Figure 2B, C). In agreement with our previous findings, the $C$. difficile burden was

104 significantly reduced by ampicillin treatment in mice reconstituted with mut BLA E. coli, but not

105 in mice reconstituted with WT BLA E. coli (Figure 2D). Direct comparison of the ampicillin-

106 treated mice confirmed a significantly higher burden in mice whose intestinal flora had the

107 capacity to hydrolyze $\beta$-lactams (Figure 2D). 
109 These findings suggest that ARGs expressed by commensal bacteria can shape the chemical

110 niche of the intestine and confer an apparent antibiotic resistant phenotype to pathogens in

111 trans, without direct acquisition of ARGs by the pathogenic microbe. We refer to this activity as

112 commensal-mediated pathogen shielding. Using two different infection models, we show that

113 production of $\beta$-lactamases, a prototypical antibiotic resistance factor, by resident intestinal

114 microbes can significantly reduce the effectiveness of ampicillin treatment, thereby generating

115 a safe environment in which otherwise sensitive pathogens are shielded from this drug.

116 Importantly, previous studies in healthy volunteers demonstrated that upon treatment with

117 cephalosporins, subjects harboring BLA-producing commensal strains, unlike BLA-negative

118 subjects, had undetectable concentrations of the drug in the feces and maintained a rich

119 microbiota, providing evidence that BLA concentrations sufficient to inactivate antibiotics are

120 commonly achieved in humans $(10,11)$.

121

122 Whether or not ARGs enrichment within the gut microbiota is detrimental to host health is a

123 complex question, and the answer is likely to be context-dependent.

124 For instance, oral administration of recombinant beta lactamase or BLA-producing bacteria was

125 shown to preserve the integrity of the microbiota following parenteral administration of beta-

126 lactam antibiotics in animal models, without affecting drug concentration in the serum (12-17).

127 These approaches were shown to be advantageous in that they preserved colonization

128 resistance against pathogens (12-17).

129 On the other hand, early work (reviewed in (18)) revealed that beta-lactamase-producing, non-

130 pathogenic bacteria, can hinder the efficacy of penicillins in vitro and in vivo, using models of 
131 subcutaneous and tonsil infection. Clinical data also suggested that the presence of one beta-

132 lactamase-producing bacterial strain at the site of infection could enhance persistence of a 133 pathogen upon antibiotic treatment (19). In these settings, members of the Bacteroides genus,

134 among the most highly represented genera in the human intestine(20), were also identified as

135 BLA-carriers.

136 Consistent with these observations, our laboratory recently showed that a few bacterial strains,

137 out of the dozens composing the microbiota of a mouse colony treated with ampicillin for over

1388 years, had the capacity to hydrolyze ampicillin, while the other bacterial strains, in isolation,

139 remained sensitive to ampicillin and thus were protected in trans by a minor subset of the

140 microbiota (21)(see Figure 4A in (22)).

142 In conclusion, we propose that commensal-mediated pathogen shielding can impair the

143 effectiveness of some antibiotic treatments during infection. While pharmacokinetic studies

144 have generally focused on antibiotic absorption, distribution, enzymatic modification, protein

145 binding and biliary/renal clearance, the role of microbiota-mediated antibiotic degradation in

146 the gut lumen and its potential for dramatically impacting responses to antibiotic treatment has

147 received less attention. Our findings extend the recently uncovered broad capacity of the gut

148 microbiota to metabolize drugs, affecting their efficacy $(23,24)$. Within this model, antibiotics

149 represent an additional class of xenobiotics that commensals can metabolize.

150 Our study suggests that presence or absence of commensal bacterial strains that inactivate

151 beta-lactam antibiotics is likely to impact clinical responses to antibiotic treatment, possibly

152 contributing to inter-individual variability in therapy outcomes. Furthermore, the occurrence of 
bioRxiv preprint doi: https://doi.org/10.1101/870931; this version posted December 10, 2019. The copyright holder for this preprint (which was not certified by peer review) is the author/funder, who has granted bioRxiv a license to display the preprint in perpetuity. It is made available under aCC-BY-NC-ND 4.0 International license.

153 pathogen shielding might be a relevant element to consider in the engineering of probiotic 154 bacterial strains to be employed in clinical practice.

155

156

157 


\section{Figure captions}

Figure 1. $\beta$-lactamase production by a model commensal curtails the efficacy of ampicillin

against Listeria monocytogenes. A) Schematic representation of the experimental design. B)

161 Nitrocefin assay performed on resuspended fecal pellets obtained from the depicted groups of

162 mice. Each well represents a different mouse, one representative experiment of three shown.

163 C) Reconstitution levels for the depicted E. coli strains as measured by plating fecal pellets on

164 day 7 post-reconstitution (day of infection) onto selective plates ( $n=8-10$, data pooled from 2

165 independent experiments, shown are individual data points and geometric mean).

D) Luminal $L m$ burden in the depicted mice 1 day post infection, measured by plating fecal

167 pellets onto selective plates. E) $L m$ burden in the depicted compartments at day 3 post

168 infection (D,E: $n=12$, data pooled from 3 independent experiments, shown are individual data

169 points and geometric mean. Mann-Whitney test: $*=p<0.05, * *=p<0.01, * * *=p<0.001)$. F)

170 Individual Lm colonies (28) or WT BLA E. coli colonies (4) from 4-5 different mice were

171 inoculated into $\mathrm{BHI}+/$ - ampicillin. OD was measured after o.n. culture. G) Colonies utilized for

172 the experiment depicted in F) were also subjected to PCR with primers specific for the TME-1 $\beta$ -

173 lactamase gene or p60 ( $\mathrm{Lm}$ positive control). Shown are results for $6 \mathrm{Lm}$ colonies and $1 \mathrm{E}$. coli

174 colony; identical results were obtained for all tested colonies. H) The cecal content of WT mice

175 reconstituted with either WT or mut BLA E. coli and administered ampicillin in drinking water

176 for two days was serially diluted and inoculated with $\mathrm{Lm}$. Lm growth was assessed after o.n.

177 culture by measurement of OD and direct plating (one representative plate shown on the left).

178 Plotted values correspond to the first dilution allowing for detectable $\mathrm{Lm}$ growth (with 1 
179 indicating $\mathrm{Lm}$ growth at all dilutions) ( $\mathrm{n}=3$, shown is mean \pm SD). Similar results were obtained utilizing antibiotic-treated, E. coli-reconstituted animals (see Supplementary Figure 1). assessed by selective plating of fecal pellets $(n=14$, data pooled from 2 independent

1913 independent experiments, shown are individual data points and geometric mean; Kruskal-

192 Wallis test with multiple comparisons: $*=p<0.05, * *=p<0.01)$.

Supplementary Figure 1. Confirmation of differential anti-listerial activity of cecal contents in

195 WT BLA vs mut BLA E. coli-reconstituted WT mice. C57BI/6 mice were treated with a

196 combination of metronidazole, neomycin and vancomycin for 3 days and reconstituted with

197 either WT or mut BLA E. coli. 7 days following reconstitution mice were administered ampicillin

198 in drinking water for 2 days, prior to sacrifice. $L m$ was cultured in serial dilutions of the cecal

199 contents, and growth was assessed after o.n. incubation. To account for variability across

200 experiments, $\mathrm{Lm}$ inhibition calculated as 1 /first cecal dilution allowing $\mathrm{Lm}$ growth was 
normalized so that the average value for WT BLA ceca would be equal to 1 in each experiment

202 ( $n=9-10$ mice from 3 different experiments, shown is mean \pm SD, Mann-Whitney test:

$203 *=p<0.05)$.

204

205 Supplementary Figure 2. Ampicillin sensitivity of $C$. difficile. C. difficile was grown to stationaty

206 phase and inoculated in medium with different concentrations of ampicillin. Shown is OD600

207 measured after o.n. incubation at $37^{\circ} \mathrm{C}$ in anaerobic conditions ( $n=3$ technical replicates).

209 Methods

210 Mouse Husbandry

211 All experiments using wild-type mice were performed with C57BL/6J female mice that were 6-8

212 weeks old; mice were purchased from Jackson Laboratories. Germ-free (GF) mice were bred in-

213 house in germ-free isolators. Following reconstitution mice were housed in sterile, autoclaved

214 cages with irradiated food and acidified, autoclaved water. All animals were maintained in a

215 specific-pathogen-free facility at Memorial Sloan Kettering Cancer Center Animal Resource

216 Center. Experiments were performed in compliance with Memorial Sloan-Kettering Cancer

217 Center institutional guidelines and approved by the institution's Institutional Animal Care and

218 Use Committee.

\section{Generation of $E$. coli strains}

221 Plasmids encoding for WT TEM-1 ßlactamase (pDIMC8-TEM1) or mutated TEM-1 ßlactamase

222 (pDIMC8-TEM1 W208G) were extracted from the RH06 and RH09 E. coli strains, published 
223 elsewhere (6), gel-purified and utilized for transformation of Stellar competent cells (Takara

224 Bio) according to manufacturer's instructions. The resulting strains were utilized for

225 experiments throughout this study. Of note, the plasmids conferred resistance to

226 chloramphenicol, and while expression of the TEM-1 gene was placed under the regulation of a

227 tac promoter, we did not induced it by IPTG treatment, but rather exclusively relied on leaky

228 transcription of the gene, to produce more physiologically-relevant conditions.

\section{Antibiotic treatment, reconstitution and infections}

231 GF mice were gavaged with either of two strains of $E$. coli, encoding for a functional or a point-

232 mutated version of TEM-1 $\beta$-lactamase, respectively. 1 week post reconstitution mice were

233 gavaged with $10^{9}$ CFUs of L. monocytogenes $(\mathrm{Lm})$ strain $10403 \mathrm{~s}$ and administered $1 \mathrm{mg}$ of

234 ampicillin (Fisher) by oral gavage daily for 2 consecutive days. Animals were euthanized at day 3

235 post infection. Reconstitution of WT mice with E. coli strains for in vitro experiments involving

236 dilution of cecal content, mice were treated for 3 days with metronidazole and vancomycin in

237 drinking water $(0.5 \mathrm{~g} / \mathrm{l})$, left on regular water for 1 day, and then gavaged with the appropriate

238 E. coli strain. 1 week post reconstitution mice were treated with ampicillin in drinking water

$239(0.5 \mathrm{~g} / \mathrm{l})$ for two days prior to being euthanized.

240 For $C$. difficile infection experiments, WT C57BI/6 mice were administered a combination of

241 metronidazole, neomycin and vancomycin (0.25 g/l each) in drinking water for 3 days, and $24 \mathrm{~h}$

242 post antibiotic regimen cessation were injected i.p. with clindamycin (200 $\mu \mathrm{g})$. On the following

243 day mice were reconstituted with either WT or mut BLA E. coli (5×10 4 CFUs) and 200-500 spores

244 of C. difficile strain VPI10463 (ATCC \#43255). 


\section{CFUs enumeration and Selective plating}

247 L. monoctogenes was identified through plating of serial dilutions of homogenized organs

248 (prepared as described elsewhere (7)) or fecal material (resuspended $100 \mathrm{mg} / \mathrm{ml}$ in PBS) onto

249 BHI plates supplemented with streptomycin $(100 \mu \mathrm{g} / \mathrm{ml})$ and nalidixic acid $(50 \mu \mathrm{g} / \mathrm{ml})$.

250 E. coli CFUs were enumerated following plating of serial dilution of fecal material onto LB plates

251 supplemented with chloramphenicol $(50 \mu \mathrm{g} / \mathrm{ml})$. E. coli CFU numbers obtained from plating of

252 ex-GF mice at day of infection onto LB plates (not supplemented with antibiotics) yielded

253 identical numbers, indicating that plasmids carrying CM resistance cassette as well as the

254 WT/mut BLA gene were maintained even in the absence of any selective pressure.

255 For detection of $C$. difficile, fecal pellets or cecal content were resuspended in deoxygenated

256 phosphate-buffed saline (PBS), and ten-fold dilutions were plated on BHI agar supplemented

257 with yeast extract, taurocholate, L-cysteine, cycloserine and cefoxitin at $37^{\circ} \mathrm{C}$ in an anaerobic

258 chamber (Coylabs) overnight.

260 Lm culture in cecal content

261 Cecal contents were recovered from E. coli reconstituted WT or GF animals, resuspended in PBS

262 at $300 \mathrm{mg} / \mathrm{ml}$ (WT) and spun down at $3000 \mathrm{rpm}$ for 10'. Serial 1:2 dilutions of the resulting

263 supernatant were generated using PBS and $100 \mu \mathrm{l}$ of each dilution were plated in replicate in

264 flat bottom 96 well plates. An equal volume of BHI medium supplemented with streptomycin

$265(200 \mu \mathrm{g} / \mathrm{ml})$ and nalidixic acid $(100 \mu \mathrm{g} / \mathrm{ml})$ acid (to prevent growth of residual E. coli) containing

266 100-1000 CFUs Lm 10403s, was added on top. Lm for this assay was prepared by re-inoculating 
267 an overnight culture in liquid $\mathrm{BHI}$ at $37^{\circ} \mathrm{C}$ on shaker, until logarithmic phase of growth was

268 reached $(O D=0.1-0.4)$. After an overnight incubation at $37^{\circ} \mathrm{C}$, the plate was assayed by $\mathrm{OD} 600$

269 reading and individual dilutions plated onto BHI-Strep-NA plates for assessment of Lm growth.

270 Normalized inhibition index was calculated as 1/first dilution allowing for $\mathrm{Lm}$ growth, with the

271 initial dilution being 1:2 to take into account the addition of a volume of $\mathrm{BHI}$ equivalent to that

272 of the medium. For example, if the first dilution where $L m$ was detected was 1:16, the resulting

273 inhibition index would be 16. Within each experiment samples were then normalized to the

274 baseline, obtained by averaging the values obtained in the control group, represented by mice

275 reconstituted with mut BLA E. coli.

276

277 PCR

278 PCR for was carried out using the following primers: $\beta$-lactamase (fw: 5'-

279 GCTATGTGGCGCGGTATTAT-3'; rev: 5'-AAGTAAGTTGGCCGCAGTGT-3', product: 191 bp); p60

280 (fw: 5'-GCGCAACAAACTGAAGCAAAGGATGC-3'; rev: 5'- CTCGCGTTACCAGGCAAATAGATGGACG-

281 3', product: 1300 BP), using the SapphireAMp Fast PCR master mix (Takara Bio) and the

282 following conditions: $94^{\circ} \mathrm{C} \times 1^{\prime}, 30 \times\left(98^{\circ} \mathrm{C} \times 5^{\prime \prime}, 58^{\circ} \mathrm{C} \times 5^{\prime \prime}, 72^{\circ} \mathrm{C} \times 15^{\prime \prime}\right)$.

284 Acknowledgments

285 We thank Ying Taur and Peter McKenney for critical discussion of the manuscript. RH06 and

286 RH09 E. coli strains carrying the pDIMC8-TEM1 and pDIMC8-TEM1 W208G plasmids were a kind

287 gift of Prof. Marc Ostermeier (Johns Hopkins University). This work was supported by NIH grant 
P30 CA008748 (to MSKCC), Burroughs Wellcome Fund Investigator in the Pathogenesis of

Infectious Disease Awards (T.M.H.).

290

S.B. was supported by an Early Postdoc Mobility Fellowship from the Swiss National Science

291

Foundation (P2EZP3_159083) and an Irvington Fellowship from the Cancer Research Institute

292 (49679).

293

Competing financial interests: E.G.P. has received speaker honoraria from Bristol Myers

for reducing Clostridium difficile infection" and \#WO2017091753A1, entitled "Methods and compositions for reducing vancomycin-resistant enterococci infection or colonization" and holds patents that receive royalties from Seres Therapeutics, Inc.

1. Nikaido H. 2009. Multidrug resistance in bacteria. Annu Rev Biochem 78:119-146.

2. Rolain JM. 2013. Food and human gut as reservoirs of transferable antibiotic resistance encoding genes. Front Microbiol 4:173.

3. Jernberg C, Lofmark S, Edlund C, Jansson JK. 2007. Long-term ecological impacts of antibiotic administration on the human intestinal microbiota. ISME J 1:56-66.

4. Becattini S, Taur Y, Pamer EG. 2016. Antibiotic-Induced Changes in the Intestinal Microbiota and Disease. Trends Mol Med 22:458-478.

5. Forsberg KJ, Reyes A, Wang B, Selleck EM, Sommer MO, Dantas G. 2012. The shared antibiotic resistome of soil bacteria and human pathogens. Science 337:1107-1111.

6. Sohka T, Heins RA, Phelan RM, Greisler JM, Townsend CA, Ostermeier M. 2009. An externally tunable bacterial band-pass filter. Proc Natl Acad Sci U S A 106:10135-10140.

7. Becattini S, Littmann ER, Carter RA, Kim SG, Morjaria SM, Ling L, Gyaltshen Y, Fontana E, Taur Y, Leiner IM, Pamer EG. 2017. Commensal microbes provide first line defense against Listeria monocytogenes infection. J Exp Med 214:1973-1989.

8. Buffie CG, Jarchum I, Equinda M, Lipuma L, Gobourne A, Viale A, Ubeda C, Xavier J, Pamer EG. 2012. Profound alterations of intestinal microbiota following a single dose of 
clindamycin results in sustained susceptibility to Clostridium difficile-induced colitis. Infect Immun 80:62-73.

9. Pamer EG. 2016. Resurrecting the intestinal microbiota to combat antibiotic-resistant pathogens. Science 352:535-538.

10. Leonard F, Andremont A, Leclerq B, Labia R, Tancrede C. 1989. Use of beta-lactamase-

16. Harmoinen J, Mentula S, Heikkila M, van der Rest M, Rajala-Schultz PJ, Donskey CJ, producing anaerobes to prevent ceftriaxone from degrading intestinal resistance to colonization. J Infect Dis 160:274-280.

11. Chachaty E, Bourneix C, Renard S, Bonnay M, Andremont A. 1993. Shedding of Clostridium difficile, fecal beta-lactamase activity, and gastrointestinal symptoms in 51 volunteers treated with oral cefixime. Antimicrob Agents Chemother 37:1432-1435.

12. Gebhart D, Lok S, Clare S, Tomas M, Stares M, Scholl D, Donskey CJ, Lawley TD, Govoni GR. 2015. A modified R-type bacteriocin specifically targeting Clostridium difficile prevents colonization of mice without affecting gut microbiota diversity. MBio 6.

13. Stiefel U, Nerandzic MM, Pultz MJ, Donskey CJ. 2014. Gastrointestinal Colonization with a Cephalosporinase-Producing Bacteroides Species Preserves Colonization Resistance against Vancomycin-Resistant Enterococcus and Clostridium difficile in Cephalosporin-Treated Mice. Antimicrobial Agents and Chemotherapy 58:4535-4542.

14. Tarkkanen AM, Heinonen T, Jogi R, Mentula S, van der Rest ME, Donskey CJ, Kemppainen T, Gurbanov K, Nord CE. 2009. P1A recombinant beta-lactamase prevents emergence of antimicrobial resistance in gut microflora of healthy subjects during intravenous administration of ampicillin. Antimicrob Agents Chemother 53:2455-2462.

15. Stiefel U, Harmoinen J, Koski P, Kaariainen S, Wickstrand N, Lindevall K, Pultz NJ, Bonomo RA, Helfand MS, Donskey CJ. 2005. Orally administered recombinant metallobeta-lactamase preserves colonization resistance of piperacillin-tazobactam-treated mice. Antimicrob Agents Chemother 49:5190-5191. Frias R, Koski P, Wickstrand N, Jousimies-Somer H, Westermarck E, Lindevall K. 2004. Orally administered targeted recombinant Beta-lactamase prevents ampicillin-induced selective pressure on the gut microbiota: a novel approach to reducing antimicrobial resistance. Antimicrob Agents Chemother 48:75-79.

17. Stiefel U, Pultz NJ, Harmoinen J, Koski P, Lindevall K, Helfand MS, Donskey CJ. 2003. Oral administration of beta-lactamase preserves colonization resistance of piperacillintreated mice. J Infect Dis 188:1605-1609.

18. Brook I. 2009. The role of beta-lactamase-producing-bacteria in mixed infections. BMC Infect Dis 9:202.

19. Brook I. 2004. Beta-lactamase-producing bacteria in mixed infections. Clin Microbiol Infect 10:777-784.

20. Human Microbiome Project C. 2012. Structure, function and diversity of the healthy human microbiome. Nature 486:207-214.

21. Kelsic ED, Zhao J, Vetsigian K, Kishony R. 2015. Counteraction of antibiotic production and degradation stabilizes microbial communities. Nature 521:516-519.

22. Caballero S, Kim S, Carter RA, Leiner IM, Susac B, Miller L, Kim GJ, Ling L, Pamer EG. 2017. Cooperating Commensals Restore Colonization Resistance to VancomycinResistant Enterococcus faecium. Cell Host Microbe 21:592-602 e594. 
bioRxiv preprint doi: https://doi.org/10.1101/870931; this version posted December 10, 2019. The copyright holder for this preprint (which was not certified by peer review) is the author/funder, who has granted bioRxiv a license to display the preprint in perpetuity. It is made available under aCC-BY-NC-ND 4.0 International license.

23. Zimmermann M, Zimmermann-Kogadeeva M, Wegmann R, Goodman AL. 2019. Mapping human microbiome drug metabolism by gut bacteria and their genes. Nature 570:462-467.

24. Zimmermann M, Zimmermann-Kogadeeva M, Wegmann R, Goodman AL. 2019. Separating host and microbiome contributions to drug pharmacokinetics and toxicity. Science 363. 
A

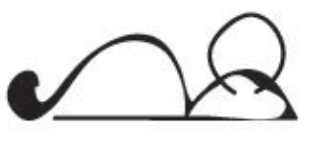

WT mice

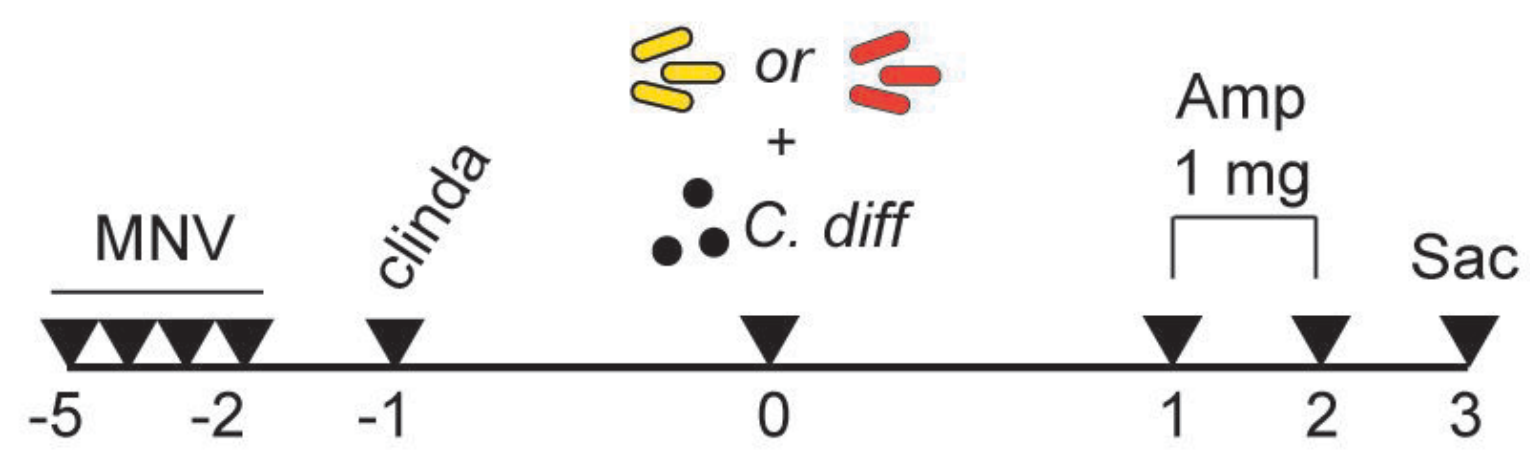

B

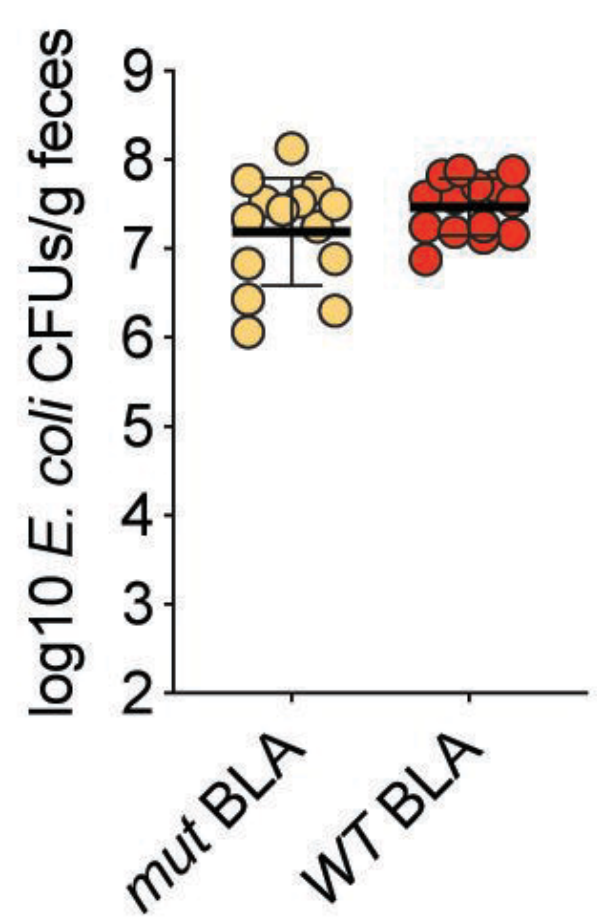

C

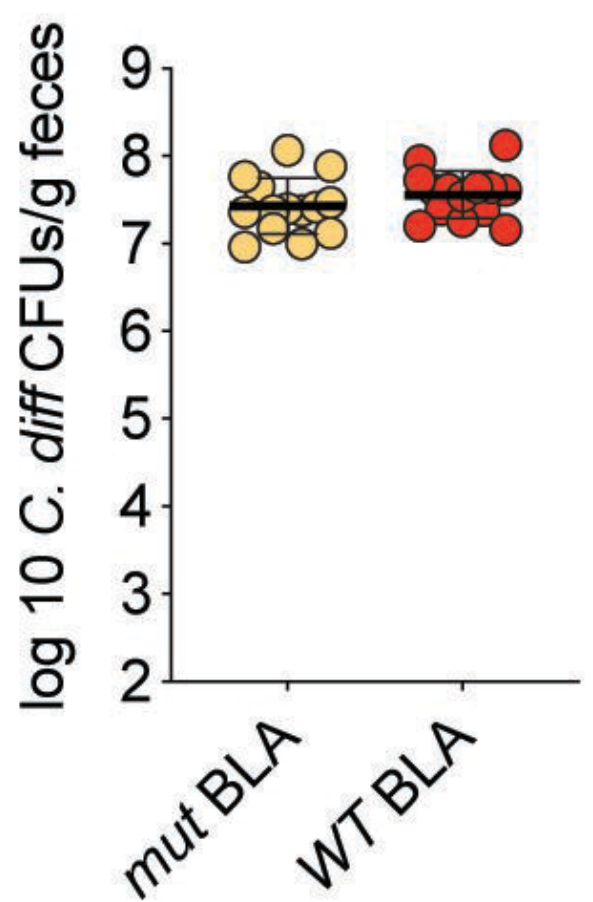

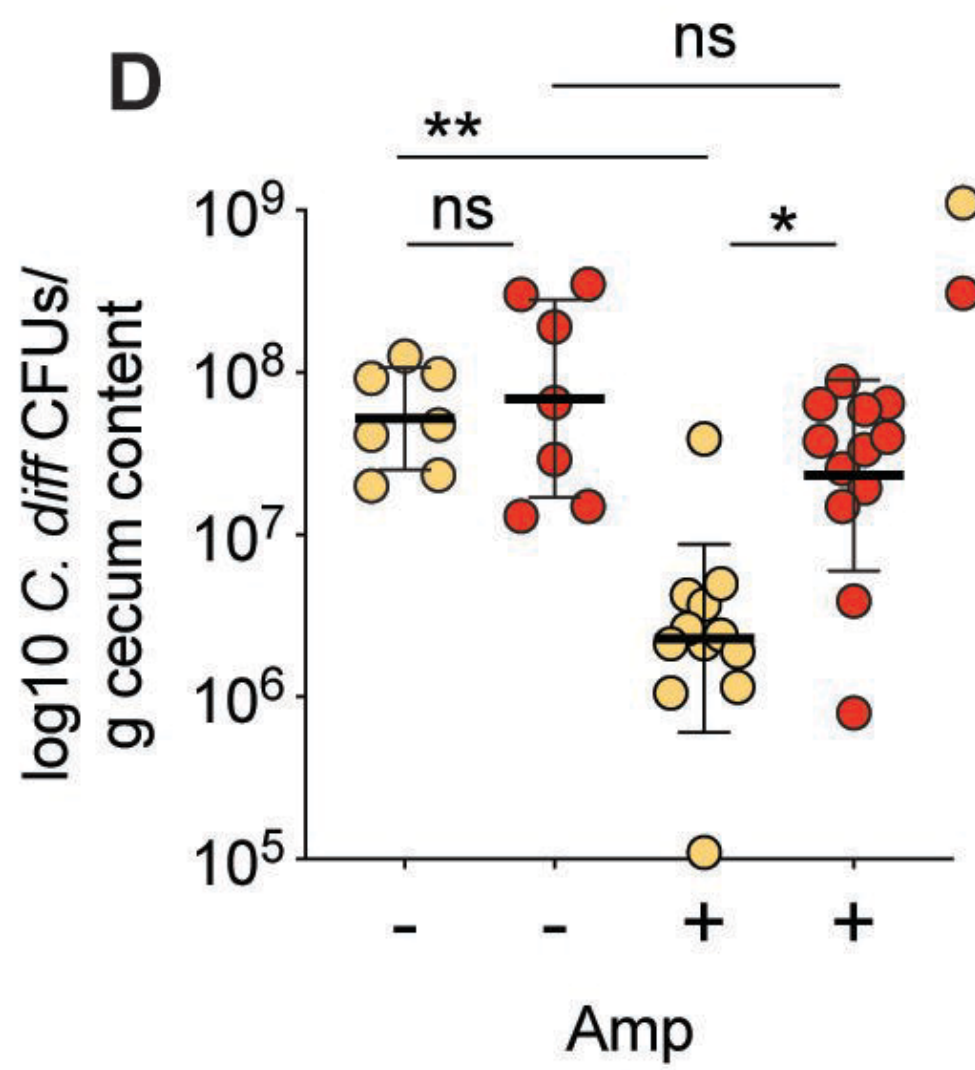

O mut BLA

- WT BLA 

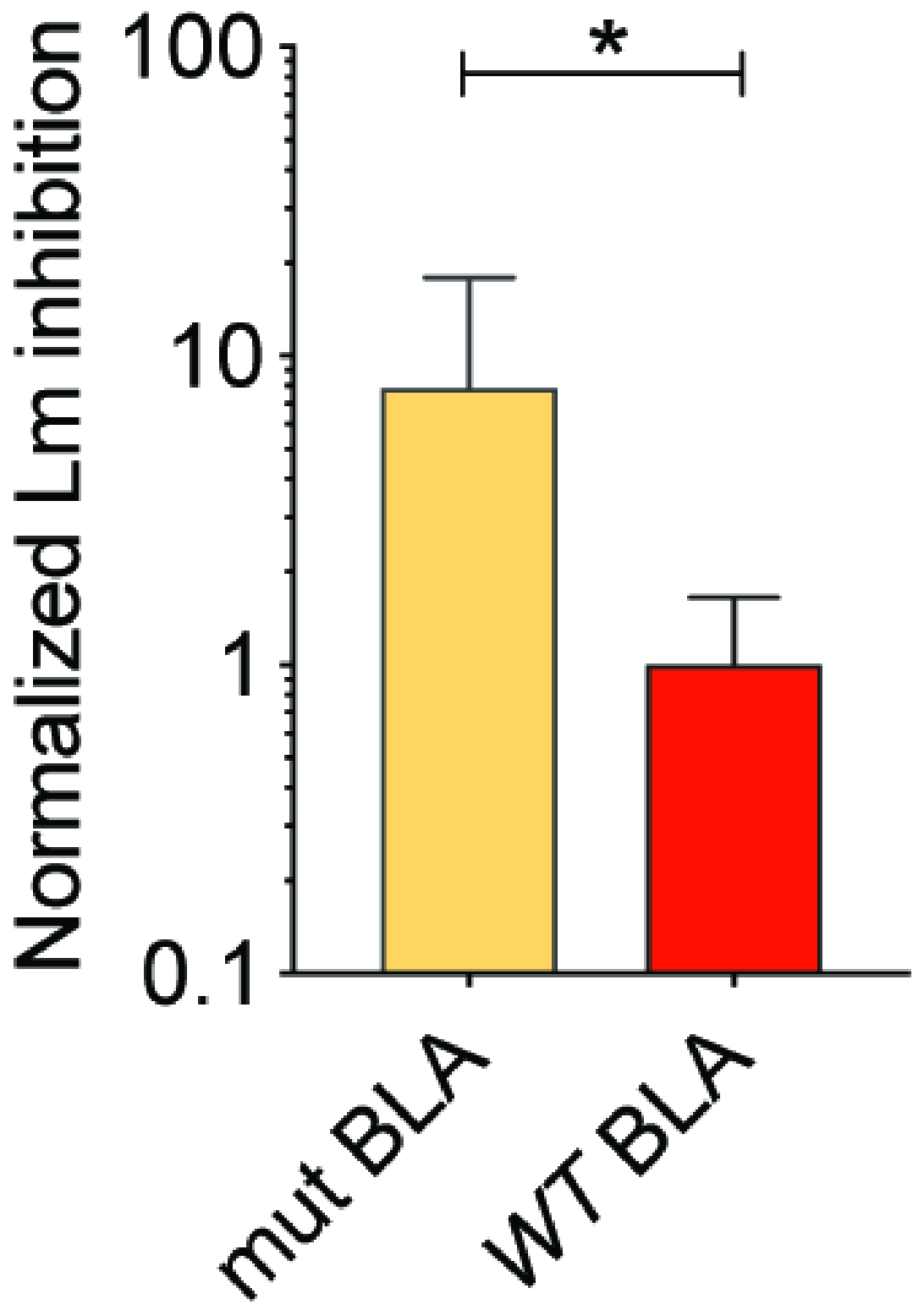
bioRxiv preprint doi: https://doi.org/10.1101/870931; this version posted December 10, 2019. The copyright holder for this preprint (which was

Supplementary Figure 2

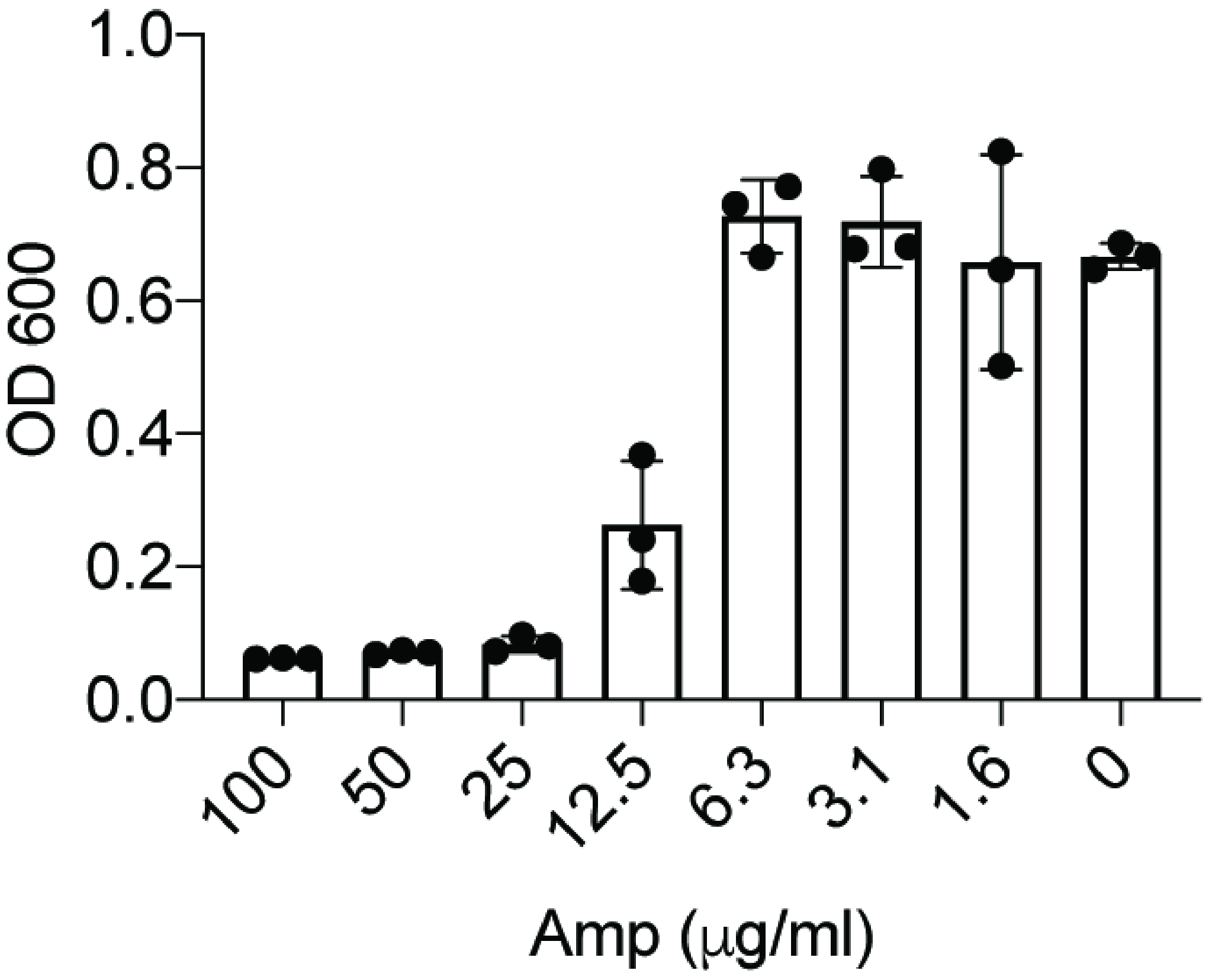

